\title{
Toxicidade de espécies vegetais sobre Callosobruchus maculatus (Coleoptera: Bruchidae) ${ }^{1}$
}

\author{
Antonia Mirian Nogueira de Moura Guerra ${ }^{2}$, Lucas Barbosa dos Santos ${ }^{3}$, Priscila Santos Silva ${ }^{4}$, Deyse \\ Silva dos Santos ${ }^{5}$
}

\begin{abstract}
Resumo: A fim de encontrar meios menos tóxicos ao meio ambiente e a saúde humana, o uso de espécies vegetais tem despontado como uma alternativa eficiente no controle de pragas de grãos armazenados. $\mathrm{O}$ caruncho, Callosobruchus maculatus (F.), é uma das principais pragas do armazenamento de feijão-caupi (Vigna unguiculata L.). O objetivo com esse estudo foi avaliar a toxicidade de pós das espécies vegetais de Caesalpinia pyramidalis, Curcuma longa L., Origanum vulgare L. e Xylopia aromatica sobre a mortalidade C. maculatus. As unidades experimentais foram constituídas por placas de Petri $(9,0 \times 1,5 \mathrm{~cm})$, com o fundo coberto por papel filtro contendo $0,5 \mathrm{~g}$ de cada pó vegetal para $20 \mathrm{~g}$ de grãos de feijão-caupi. Foram estimados os tempos letais para 10, 30, 50, $70 \mathrm{e}$ $90 \%$ dos adultos do inseto. Utilizou-se 4 repetições. Em todas as espécies foi constatada mortalidade nas primeiras $6 \mathrm{~h}$ de contato com os extratos, com maior expressão para C. longa. Após 24h de exposição foram contatadas mais de $60 \%$ de mortalidade acumulada para todas as espécies. O intervalo entre 19 e 25 horas, foi suficiente para provocar mortalidade de $60 \%$ da população conforme a espécie vegetal, e para ocasionar $90 \%$ de mortalidade foram necessárias entre 72 a 90 horas de exposição aos pós das diferentes espécies. O açafrão ocasionou $50 \%$ de mortalidade acumulada com $18 \mathrm{~h} 36$ ' de exposição, sendo a espécie mais eficiente no controle de adultos de $C$. maculatus, demonstrando a capacidade desta espécie para o desenvolvimento de produtos com fins inseticidas.
\end{abstract}

Palavras-Chave: Caesalpinia pyramidalis, Curcuma longa L., praga do feijão.

\section{Toxicity of plant species on Callosobruchus maculatus (Coleoptera: Bruchidae)}

\begin{abstract}
In order to find ways that are less toxic to the environment and human health, the use of plant species has emerged as an efficient alternative in the control of stored grain pests. The carouscho, Callosobruchus maculatus (F.), is one of the main pests of the storage of cowpea (Vigna unguiculata L.). The objective of this study was to evaluate the toxicity of plant species of Caesalpinia pyramidalis, Curcuma longa L., Origanum vulgare L. and Xylopia aromatica on C. maculatus mortality. The experimental units were composed of Petri dishes $(9.0 \times 1.5 \mathrm{~cm})$, with the bottom covered by filter paper containing $0.5 \mathrm{~g}$ of each vegetable powder for $20 \mathrm{~g}$ of cowpea beans. Lethal times were estimated for 10, 30, 50, 70 and $90 \%$ of insect adults. Four replicates were used. Mortality was observed in all species in the first 6 hours of contact with the extracts, with higher expression for C. longus. After 24 hours of exposure, more than $60 \%$ of accumulated mortality was contacted for all species. The interval between 19 and 25 hours was sufficient to cause mortality of $60 \%$ of the population according to the plant species, and to cause $90 \%$ of mortality, it was necessary between 72 and 90 hours of exposure to the different species. Saffron caused 50\% of accumulated mortality with $18 \mathrm{~h} 36$ 'of exposure, being the most efficient species in the control of adults of $C$. maculatus, demonstrating the capacity of this species for the development of insecticidal products.
\end{abstract}

Key words: Caesalpinia pyramidalis, Curcuma longa L., pest of the bean.

\footnotetext{
${ }^{1}$ Submetido em 27/09/2018 e a provado em 03/01/2019

${ }^{2}$ Doutora em Fitotecnia; Professora, Universidade Federal do Oeste da Bahia (UFOB), Centro Multidisciplinar de Barra, Barra-BA, CEP: 47.100-000; E-mail: mirianagronoma@ @otmail.com.br

${ }^{3}$ Graduando em Agronomia, Universidade Federal do Oeste da Bahia (UFOB), Centro Multidisciplinar de Barra, Barra-BA, CEP: 47.100-000; E-mail: lukkas@gmail.com

${ }^{4}$ Graduanda em Agronomia, Universidade Federal do Oeste da Bahia (UFOB), Centro Multidisciplinar de Barra, Barra-BA, CEP: 47.100-000; E-mail: prissssilva@gmail.com

${ }^{5}$ Graduanda em Agronomia, Universidade Federal do Oeste da Bahia (UFOB), Centro Multidisciplinar de Barra, Barra-BA, CEP: 47.100-000; E-mail: deyse@ hotmail.com
} 


\section{Introdução}

O feijão-caupi, feijão-de-corda ou feijãomacassar (Vigna unguiculata (L.) Walp.) é uma excelente fonte de proteínas (23-25\%) e apresenta todos os aminoácidos essenciais, carboidratos (62\%), vitaminas e minerais, além de possuir grande quantidade de fibras dietéticas, baixa quantidade de gordura (teor de óleo de $2 \%$ ) e não conter colesterol. Representa alimento básico para as populações principalmente do Norte e Nordeste brasileiro (Vale et al., 2017). Pelo seu valor nutritivo, o feijão-caupi é cultivado principalmente para a produção de grãos, secos ou verdes, visando o consumo humano in natura, na forma de conserva ou desidratado. Além disso, também é utilizado como forragem verde, feno, ensilagem, farinha para alimentação animal e, ainda, como adubação verde e proteção do solo (Vale et al., 2017).

Considerando-se a metodologia da FAO (Organização das Nações Unidas para a Alimentação e a Agricultura), a produção mundial média anual de feijão-caupi, entre 2007 a 2011, foi de 5,6 milhões de toneladas. O principal país produtor de caupi é a Nigéria que responde por $48 \%$ da produção mundial. Em seguida vem o Níger, com $24 \%$, e em terceiro Burkina Faso com 8\%. As três nações respondem por $80 \%$ da produção mundial de feijão-caupi seco (Vale et al., 2017).

Segundo a CONAB (Companhia Nacional de Abastecimento) na safra 2016/17, o feijão-caupi foi o terceiro tipo mais cultivado no país, na primeira safra. A área e a produtividade total de feijão-caupi cresceram cerca de $18 \%$ e $119 \%$, respectivamente, devido as condições climáticas mais favoráveis para o cultivo dessa leguminosa. O quadro climático apresentou-se favorável e a produção foi superior em $158 \%$, alcançando 210,9 mil toneladas. O cultivo de feijão-caupi é praticado na $1^{\mathrm{a}}$ safra nas regiões Nordeste e Norte e na $2^{\mathrm{a}}$ safra nestas regiões além da região CentroOeste. Os estados do Ceará (98.200 t), Piauí $(44.600 \mathrm{t})$ e Pernambuco $(43.400 \mathrm{t})$ foram os maiores produtores em $1^{\text {a }}$ safra e o estado Mato Grosso apresentou produção de $107.000 \mathrm{t}$ na $2^{\mathrm{a}}$ safra e a produtividade média nacional foi de 400 $\mathrm{kg} \mathrm{ha}^{-1}$.

As principais espécies de insetos que atacam o feijão na fase pós-colheita são os bruchideos Acanthoscelides obtectus (Say), Callosobruchus maculatus (F.) e Zabrotes subfasciatus (Boh.). Dentre estes, o $C$. maculatus destaca-se como a principal praga de grãos de feijão-caupi, durante o armazenamento. Estes insetos ocasionam danos como a redução da massa e/ou volume; a redução na qualidade fisiológica e na capacidade de germinação; e o aumento da temperatura e do teor de água dos grãos (Procópio et al., 2003).

$O$ controle de insetos-praga tem sido intensificado nas últimas décadas, sendo utilizados inseticidas protetores, piretróides e organofosforados e o fumigante fosfeto de alumínio. Todavia, o uso contínuo e indiscriminado de inseticidas, para o tratamento de produtos alimentícios, tem sido questionado em todo o mundo (Faroni et al., 2009).

Observa-se uma crescente preocupação da sociedade com relação aos níveis de resíduos dos inseticidas presentes nos alimentos e os riscos oferecidos por eles à saúde dos consumidores. Diante disto, surge a necessidade de estudos sobre técnicas alternativas de proteção e conservação de produtos armazenados, destacando-se 0 armazenamento hermético e o uso de inseticidas botânicos.

No que diz respeito aos inseticidas botânicos, destaca-se que são produtos de origem vegetal, que apresentam algum efeito tóxico sobre insetos. Alguns destes produtos têm sido utilizados ao longo de gerações para o controle de pragas, sendo fruto do conhecimento empírico de comunidades tradicionais em diversos países. Normalmente são usados na forma de pós, extratos ou óleos, os quais são fáceis de serem obtidos e, de um modo geral, inócuos para os aplicadores e consumidores (Vieira et al., 2004; Vieira et al., 2001).

Estudos têm sido conduzidos em vários países, a fim de identificar compostos vegetais que possam ser utilizados no combate a pragas. A este respeito, já foram identificados mais de 100.000 metabólitos secundários com propriedades inseticidas, como os alcalóides, os terpenóides, os flavonóides e as quinonas, em aproximadamente 200.000 espécies de plantas em todo o mundo (Potenza et al., 2004; Procópio et al., 2003). Estes compostos apresentam múltiplos modos de ação sobre os insetos, destacando-se: toxicidade aguda, repelência, inibição da alimentação, crescimento, desenvolvimento e reprodução (Dequech et al., 2008). Todavia, o uso de 
inseticidas botânicos para controle e manejo de insetos-praga de grãos armazenados tem sido limitado.

Espécies vegetais do gênero Azadirachta, Chenopodium, Datura, Jatropha, Parthenium e Phytoloca, entre outros, possuem comprovado efeito tóxico sobre diferentes espécies de insetos, incluindo pragas de produtos armazenados (Coitinho et al., 2011; Guerra et al., 2009; Coitinho et al., 2006; Mazzonetto e Vendramim, 2003).

O objetivo deste trabalho foi estudar o efeito de extratos secos (pós) das espécies Caesalpinia pyramidalis Tul. (Catinga de Porco), Curcuma longa L. (Açafrão), Origanum vulgare L. (Orégano) e Xylopia aromatica (Lam.) Mart. (Pimentinha da Serra) sobre a mortalidade de adultos de $C$. maculatus, por meio de ensaios de tempo resposta.

\section{Material e Métodos}

\subsection{Criação do caruncho-do-feijão maculatus e obtenção de espécies vegetais}

Avaliou-se efeito bioinseticida das espécies vegetais: C. pyramidalis (Catinga de Porco cascas), C. longa (Açafrão - rizoma), O. vulgare (Orégano - folhas) e X. aromatica (Pimentinha da Serra - frutos).

Os insetos C. maculatus foram obtidos de criações mantidas no Laboratório da Universidade Federal do Oeste da Bahia (UFOB) campus Barra, os quais foram criados em frascos de vidro com capacidade de 1,0 L sob condições constantes de temperatura $\left(30^{\circ} \mathrm{C}\right)$ e umidade relativa $(70 \%)$ e escotofase de 24 horas. Como substrato alimentar, foram utilizados grãos de feijão-caupi inteiros com teor de água de $13 \%$ base úmida, previamente expurgados com fosfina e mantidos sob refrigeração $\left(-18{ }^{\circ} \mathrm{C}\right)$ para evitar reinfestação.

As espécies vegetais que foram avaliadas, já haviam apresentado efeito tóxico para os adultos de $C$. maculatus em ensaios de tempo resposta previamente realizados. Utilizou-se uma dose fixa de $0,5 \mathrm{~g}$, estabelecida com base em estudo anterior.

\subsection{Tratamentos}

Para a obtenção dos extratos, foi utilizado o equipamento moinho de facas para triturar as amostras secas de cada espécie vegetal.

As unidades experimentais foram constituídas por placas de Petri $(9,0 \times 1,5 \mathrm{~cm})$, com o fundo coberto por papel filtro contendo $0,5 \mathrm{~g}$ de cada extrato vegetal para $20 \mathrm{~g}$ de grãos de feijão-caupi. Em cada placa foram acondicionados 50 insetos adultos não sexados, sendo as placas posteriormente fechadas e mantidas em câmara climática do tipo B.O.D., à temperatura de $30^{\circ} \mathrm{C}$ e umidade relativa de $70 \%$, até a avaliação da mortalidade dos insetos. $\mathrm{O}$ controle foi constituído por placas de Petri contendo apenas os insetos, sem qualquer tratamento com extrato. A mortalidade dos insetos foi avaliada após 24 horas e se estendendo até 96 horas após a montagem dos bioensaios.

\subsection{Avaliação e obtenção dos dados}

Avaliou-se a variação do tempo de exposição do extrato seco (pó) sobre a mortalidade dos insetos, considerando um intervalo de 96 horas. Nas primeiras 24 horas foram realizadas avaliações em intervalos de 6 horas, após isto, as avaliações transcorreram a cada 24 horas. Os insetos foram considerados mortos quando tocados por pinça e não foi observado movimento. Foram estimados os tempos letais para 10, 30, 50, 70 e $90 \%$ dos adultos do inseto, em relação a cada espécie identificada como tóxica.

\subsection{Delineamento e análise estatística}

O delineamento usado foi o inteiramente casualizado (DIC) com quatro repetições para cada tratamento e os dados obtidos foram submetidos à análise de variância e ajustados modelos matemáticos.

\section{Resultados e Discussão}

Em geral, todas as espécies apresentaram efeito sobre os insetos nas primeiras 24h, momento em que foi constatada a maior ocorrência de mortalidade para todas as espécies vegetais analisadas, no entanto, $X$. aromatica e Caesalpinia pyramidalis demonstraram-se como as mais eficientes (Figura 1).

Nas primeiras 6 horas de exposição foram observados os efeitos deletérios dos extratos, sendo que $C$. longa sobressaiu-se entre os demais, 
causando cerca de $23 \%$ da mortalidade dos insetos; em seguida, houve um decréscimo entre 12 e 18 horas (aproximadamente 8\%) e um outro pico da mortalidade às 24 horas $(31 \%$ de mortalidade), no entanto, ocorreu um decréscimo, porém as mortalidades ocorreram de forma constante até às 96 horas de observação (Figura $1)$.

As espécies $O$. vulgare e $X$. aromatica apresentaram resultados semelhantes, sendo que nas primeiras 6 horas ambas atingiram cerca de $18 \%$ de mortalidade e entre 12 e 96 horas embora tenha apresentado um decréscimo. Às $24 \mathrm{~h}$ ocorreram picos de mortalidade cerca de $38 \%$ e $43 \%$, respectivamente, e a pimentinha da serra se destacou às $24 \mathrm{~h}$ causando a maior aproximadamente $44 \%$ de mortalidade (Figura 1). Por outro lado, C. pyramidalis nas primeiras 6 horas foi a menos eficiente que as outras espécies, com apenas $12 \%$ da mortalidade, observaram-se que a partir das 18 horas ocorreu um incremento na mortalidade em 24 horas foi alcançado o pico com aproximadamente $41 \%$, sendo a segunda espécie com a proporcionar maior mortalidade nesse período (Figura 1).

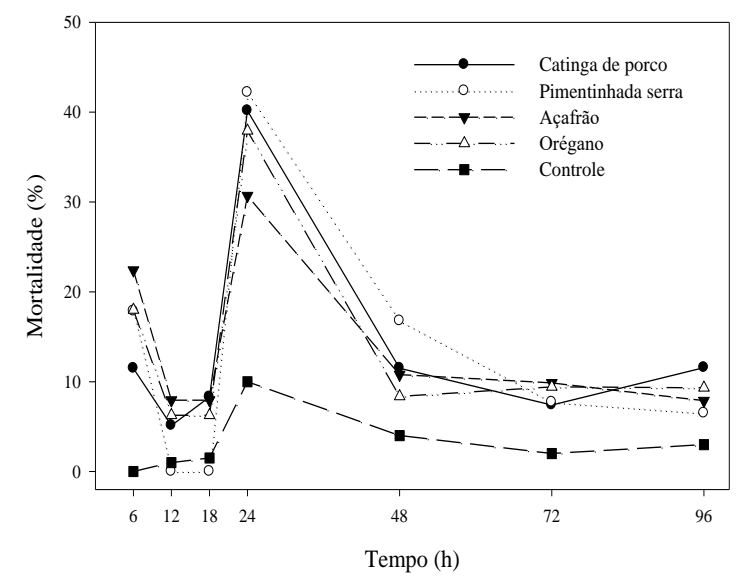

Figura 1 Mortalidade de adultos de Callosobruchus maculatus (F.) na dose 0,5g de pós de Caesalpinia pyramidalis Tul. (Catinga de Porco), Curcuma longa L. (Açafrão), Origanum vulgare L. (Orégano) e Xylopia aromatica (Pimentinha da Serra) para cada 20 g de feijão-caupi.

Seguindo a mesma linha de pesquisa, diversos autores demonstraram em seus trabalhos a ação eficaz dos pós vegetais sobre a mortalidade de gorgulhos durante o armazenamento. Procópio et al., (2003) analisando o efeito tóxico do pó da planta Chenopodium ambrosioides (Matruz) sobre a fase adulta do $Z$. subfasciatus, verificaram mortalidade de $100 \%$ e uma DL50 de $0,018 \mathrm{~g}$ de pó para cada $20 \mathrm{~g}$ de grãos de feijão comum (Phaseolus vulgaris).

O extrato de $C$. pyramidalis apresentou maior eficiência de mortalidade em comparação ao controle, sendo que entre 6 e $18 \mathrm{~h}$ a mortalidade acumulada demonstrou um aumento gradativo (16 e 23\%, respectivamente). $\mathrm{O}$ ápice de mortalidade acumulada foi às $96 \mathrm{~h}$ com cerca de 95\%, contudo, a melhor eficiência de controle em apenas $24 \mathrm{~h}$ com aproximadamente $65 \%$ de mortalidade dos adultos de $C$. maculatus (Figura 2A). Para X. aromatica, entre às 6 e 18h não foram observados incrementos na mortalidade acumulada, sendo que nas primeiras 6 horas ocorreu cerca de $19 \%$, enquanto que no controle todos os indivíduos permaneceram vivos (Figura 2B). Entre 18 e $24 \mathrm{~h}$ foi alcançada aproximadamente $61 \%$ de mortalidade acumulada. Ao final das avaliações foi obtido mais de $90 \%$ de mortalidade.

A espécie $X$. aromatica pertencente à família Annonaceae, conhecida também por produzir metabólitos secundários que podem ter efeito toxicológico sobre algumas espécies de insetos. Os dados do presente estudo corroboram com pesquisas já realizadas com insetos que causam prejuízos durante o armazenamento de grão, especialmente aqueles da ordem Coleóptera, nos quais várias espécies de anonáceas apresentaram um efeito fumigante, como por exemplo $C$. maculatus controlados com Annona muricata (Adeoye e Ewete, 2010); Capsicum chinensis com Annona senegalensis (Kotkar et al., 2002). Segundo trabalho realizado por Silva Neto (2015), foi constatado que o pó de Piper tuberculatum Jacq (Pimenta de macaco) nas doses de 0,049 e 0,086 kg reduziram considerável o número ovos viáveis, deixando nítido o efeito ovicida que a espécie apresenta sobre a oviposição do inseto nos grãos de feijão-fava.

Dentre todas as espécies estudadas, $C$. longa e $O$. vulgare foram as que apresentaram melhor eficiência sobre a mortalidade acumulada dos insetos adultos, onde nas primeiras $6 \mathrm{~h}$ de contato foram observadas aproximadamente $23 \%$ e $19 \%$ de mortalidade, respectivamente, e entre 6 e $18 \mathrm{~h}$ foi observado um aumento de $16 \%$ e $13 \%$ sobre as respectivas mortalidades acumuladas (Figura 3). Ainda nesse sentido, com $24 \mathrm{~h}$ de exposição 
observou-se cerca de 74\% (Figura 3A) e 70\% (Figura 3B) de mortalidades acumuladas de adultos de C. maculatus.

O açafrão é uma planta pertencente à família Zingiberaceae em que o rizoma é a parte mais utilizada por possuir características marcantes como odor típico, aroma picante e sabor amargo que o classificam como especiaria, conferido pela curcumina, pigmento responsável pela pigmentação amarela (Péret-Almeida et al., 2006).

Em experimentos realizados por Gott et al. (2009) em que se trabalharam com ensaios de repelência com óleo essencial de $C$. longa sobre uma das principais pragas de milho armazenado, Sitophilus zeamais, constatou-se sua eficiência como repelente sobre o $S$. zeamais, sendo que o mesmo tem a ação diminuída com o passar do tempo. Ainda segundo Gott et al., (2009) a diminuição da ação do óleo essencial se dá porque que os compostos responsáveis pela atividade repelente vão perdendo tal característica, provavelmente por volatilização, tendo em vista que os constituintes desse óleo essencial são bastante voláteis. A curcumina apresenta-se como um dos princípios ativos em Açafrão, composto com comprovada ação antimicrobiana (Souza et al., 2010). Desse modo, atribuímos efeito sobre a mortalidade do $C$. maculatus à curcumina $\mathrm{e}$ alguns outros compostos presentes na espécie. Já para o Orégano que é uma planta condimentar muito conhecida por suas propriedades antimicrobianas e antioxidantes, sendo que no seu óleo essencial são encontrados os compostos químicos timol e carvacrol, sendo constatado atividade antibacteriana sobre Escherichia coli e Staphylococcus aureus e Salmonella choleraesuis (Araújo et al., 2015).

Os tempos (letais) para a dose de $0,5 \mathrm{~g}$ para cada $20 \mathrm{~g}$ de feijão-caupi de pó das espécies açafrão, catinga de porco, orégano e pimentinha da serra que geraram mortalidades de $10 \%, 30 \%$, $50 \% 70 \%$ e $90 \%$ da população de C. maculatus, onde o intervalo de tempo de 19 a 25 horas, foi suficiente para provocar mortalidade de $50 \%$ da população conforme espécie, e para ocasionar $90 \%$ de mortalidade foram necessárias entre 72 a 90 horas de exposição aos pós das diferentes espécies (Tabela 1). Observou-se também que $C$. longa $\mathrm{L}$. foi a espécie mais eficiente que assegurou a mortalidade em menor tempo, obteve-se $50 \%$ de controle da população em $18 \mathrm{~h}$ 36 .

Substâncias potencialmente inseticidas ou repelentes são encontradas em muitas plantas, e podem ser obtidas na forma de pó, extratos e óleos essenciais. Dentre os principais compostos responsáveis por esta ação, estão os monoterpenos, citronelol, linalol, mentol e mentona (Panizzi e Parra, 1991). Os monoterpenos podem causar interferência tóxica nas funções bioquímicas e fisiológicas de insetos herbívoros, sendo que a maioria deles agem apenas como repelente. $\mathrm{O}$ efeito tóxico de um óleo essencial envolve muitos fatores, a toxina pode ser inalada, ingerida ou também absorvida pelo tegumento dos insetos (Regnault-Roger, 1997).
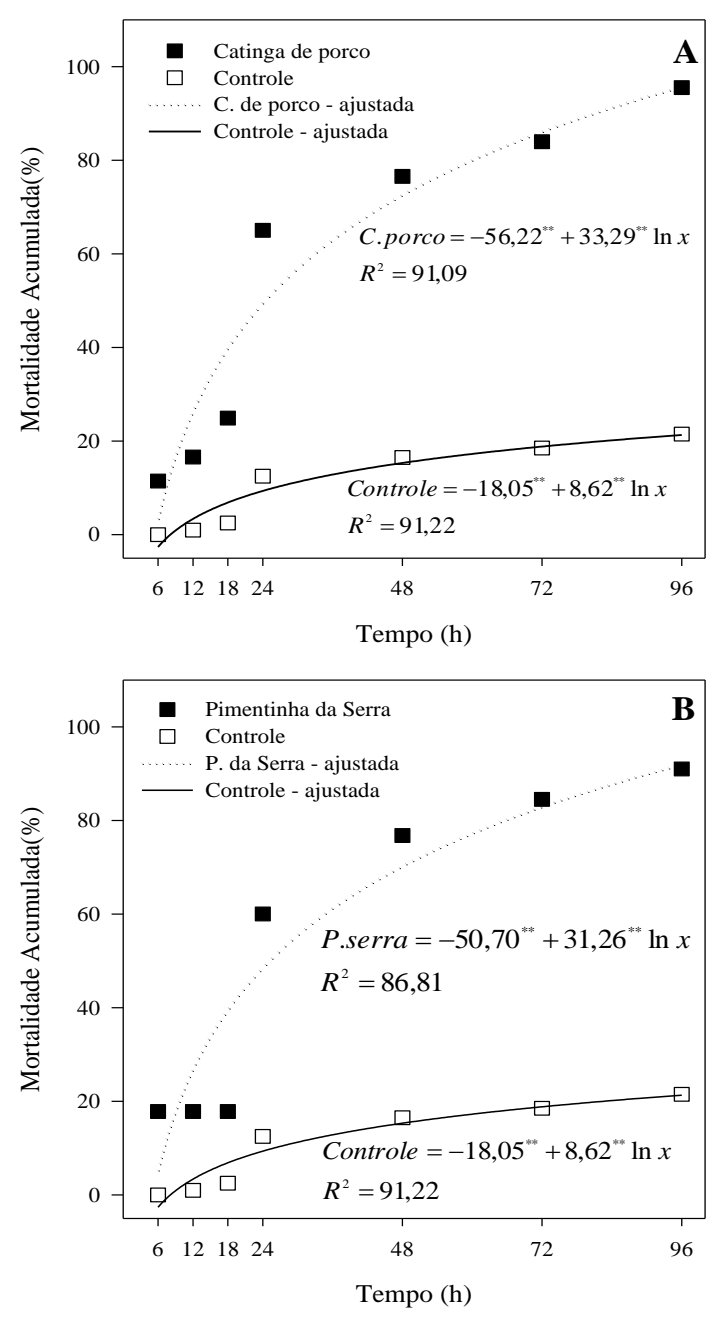

Figura 2 Mortalidade acumulada de adultos de Callosobruchus maculatus (F.) na dose 0,5g de pós de Caesalpinia pyramidalis (Catinga de Porco - A) e Xylopia aromatica (Pimentinha da Serra - B) para cada $20 \mathrm{~g}$ de feijão-caupi. 

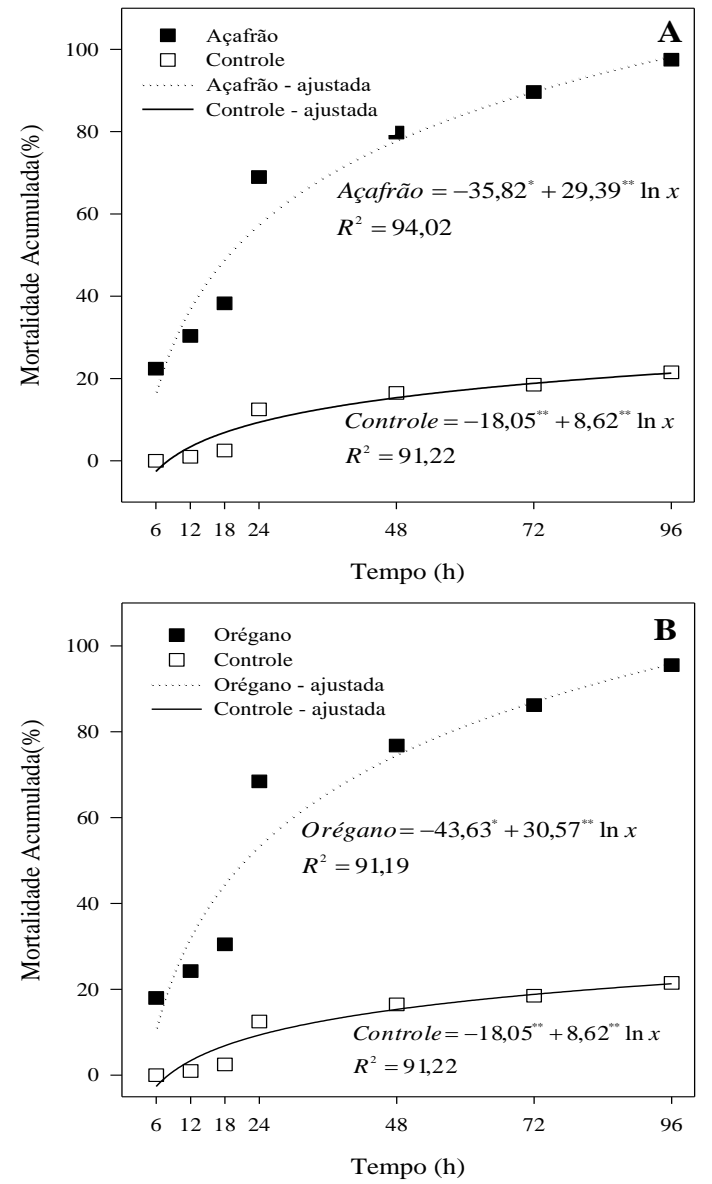

Figura 3 Mortalidade acumulada de adultos de Callosobruchus maculatus (F.) na dose $0,5 \mathrm{~g}$ de pós de Curcuma longa L. (Açafrão - A) e Origanum vulgare L. (Orégano - B) para cada $20 \mathrm{~g}$ de feijão-caupi.
Podendo ter ação de algum composto supracitado, Guerra et al. (2009) tiveram uma boa resposta testando extratos vegetais de Rosmarinus officinalis L., Peumus boldus (Molina), Matricaria chamomilla L., Baccharis trimera (Less.) DC., Camellia sinensis (L.) Kuntze, Thea sinensis L., Ilex paraguariensis St. Hil. e Pimpinella anisum L. no controle de $C$. maculatus, no qual o pó de $P$. boldus além de ter ocasionado $100 \%$ de mortalidade aos adultos, reduziu drasticamente o número de ovos (10 ovos em média), diferente do observado na testemunha (125 ovos em média).

Uma opção ao controle químico é a utilização de produtos advindos de espécies vegetais, dentre algumas formulações como os extratos vegetais secos ou aquosos (partes das plantas secas e trituradas). Esses produtos apresentam como vantagens um custo reduzido, facilidade de obtenção e utilização, mínimo conhecimento para o seu manuseio, e ainda apresentam pouco ou nenhum impacto ao ser humano e ao meio ambiente (Mazzonetto e Vendramim, 2003). Desse modo, podemos inferir que todas as espécies aqui utilizadas apresentaram potencial para controle do $C$. maculatus.

Tabela 1 Tempos letais (em horas) de exposição de adultos de Callosobruchus maculatus (F.) a dose 0,5g de pós de Caesalpinia pyramidalis Tul. (Catinga de Porco), Curcuma longa L. (Açafrão), Origanum vulgare L. (Orégano) e Xylopia aromatica (Pimentinha da Serra) para cada $20 \mathrm{~g}$ de feijão-caupi

\begin{tabular}{|c|c|c|c|c|}
\hline \multirow{2}{*}{ Mortalidade populacional (\%) } & Açafrão & Catinga de porco & Orégano & Pimentinha da Serra \\
\hline & \multicolumn{4}{|c|}{ Tempo letal (h) } \\
\hline 10 & $4 \mathrm{~h} 48^{\prime}$ & $7 \mathrm{~h} 18^{\prime}$ & $5 \mathrm{~h} 48^{\prime}$ & $7 \mathrm{~h}$ \\
\hline 30 & $9 \mathrm{~h} 24^{\prime}$ & $13 \mathrm{~h} 24^{\prime}$ & $11 \mathrm{~h} 12^{\prime}$ & $13 \mathrm{~h} 30^{\prime}$ \\
\hline 50 & $18 \mathrm{~h} 36^{\prime}$ & $24 \mathrm{~h} 24^{\prime}$ & $21 \mathrm{~h} 24^{\prime}$ & $25 \mathrm{~h} 6^{\prime}$ \\
\hline 70 & $36 \mathrm{~h} \mathrm{42}$ & $44 \mathrm{~h} 24^{\prime}$ & $41 \mathrm{~h} 12^{\prime}$ & $48 \mathrm{~h} 36^{\prime}$ \\
\hline 90 & $72 \mathrm{~h} 30^{\prime}$ & $81 \mathrm{~h}$ & $79 \mathrm{~h} \mathrm{12}$ & $90 \mathrm{~h} 18^{\prime}$ \\
\hline
\end{tabular}

\section{Conclusão}

As espécies $C$. pyramidalis, C. longa, $O$. vulgare e $X$. aromatica ocasionaram mortalidade sobre $C$. maculatus nas primeiras $6 \mathrm{~h}$ de contato com dos insetos com os extratos, sendo que $C$. longa se sobressaiu em relação as demais.
Após 24h de exposição dos insetos aos extratos vegetais foram contatadas mais de $60 \%$ de mortalidade acumulada para todas as espécies.

O açafrão ocasionou $50 \%$ de mortalidade acumulada com 18h 36' de exposição, e foi a espécie mais eficiente no controle de adultos de C. maculatus. 


\section{Agradecimentos}

Ao Conselho Nacional de Desenvolvimento Científico e Tecnológico - CNPq, pela concessão da bolsa de Iniciação Científica.

\section{Referências}

Adeoye, O. T.; Ewete, F. K. Potentials of Annona muricata Linnaeus (Annonaceae) as a botanical insecticide against Callosobruchus maculatus Fabricius (Coleoptera: Bruchidae). Journal of Agriculture, Forestry and the Social Sciences, v.8, n.2, p.147-151, 2010. https://www.ajol.info/index.php/joafss/article/view/71652

Araújo, L. S.; Araújo, R. S.; Serra, J. L.; Nascimento, A. R. Composição química e susceptibilidade do óleo essencial de óregano (Origanum vulgare L., família Lamiaceae) frente à cepas de Escherichia coli, Staphylococcus aureus e Salmonella choleraesuis. Boletim do Ceppa, v.33, n.1, p.73-78, 2015. https://revistas.ufpr.br/alimentos/article/viewFile/43808/2 $\underline{6557}$

Coitinho, R. L. B. C.; Oliveira, J. V.; Gondim Júnior, M. G. C.; Câmara, C. A. G. Atividade inseticida de óleos vegetais sobre Sitophilus zeamais Mots. (Coleoptera: Curculionidae) em milho armazenado. Revista Caatinga, v.19, n.2, p.176-182, 2006. https://periodicos.ufersa.edu.br/index.php/caatinga/article /view/39

Coitinho, R. L. B. C.; Oliveira, J. V.; Gondim Júnior, M. G.C.; Câmara, C. A. G. Toxicidade por fumigação, contato e ingestão de óleos essenciais para Sitophilus zeamais Motschulsky, $1885 \quad$ (Coleoptera: Curculionidae). Ciência e Agrotecnologia, v.35, n.1, p.172-178, 2011. https://doi.org/10.1590/S1413-70542011000100022

CONAB. Acompanhamento da Safra Brasileira de Grãos - Sétimo Levantamento da Safra 2016/2017. Brasília: CONAB, 2017.

Dequech, S. T. B.; Sausen, C. D.; Lima C.G.; Egewarth, R. Efeito de extratos de plantas com atividade inseticida no controle de Microtheca ochroloma Stal (Col.: Chrysomelidae), em laboratório. Biotemas, v.21, n.1, p.41-46, 2008. https://doi.org/10.5007/2175-7925.2008v21n1p41

Faroni, L.R.A.; Alencar, E.R.; Paes, J.L.; Costa, A.R.; Roma, R.C.C. Armazenamento de soja em silos tipo bolsa. Engenharia Agrícola, v.29, n.1, p.91-100, 2009. https://doi.org/10.1590/S0100$\underline{69162009000100010}$

Gott, R. M.; Petacci, F.; Costa, M. A.; Grazziotti, G. H.; Tavares, W. S.; Freitas, S. S. Avaliação do efeito residual repelente do óleo essencial de Curcuma longa sobre Sitophilus zeamais Mots. (Coleoptera: Curculionidae). Anais... Anais do XXVIII Congresso Nacional de Milho e Sorgo, 2010, Associação Brasileira de Milho e Sorgo. IAPAR, Londrina, Paraná, 2010. http://erevista.unioeste.br/index.php/actaiguazu/article/view/143 $\underline{96}$

Guerra, A. M. N. M.; Maracajá, P. B.; Freitas, R. S.; Sousa, A. H.; Sousa, C. S. M. Atividade inseticida de plantas medicinais sobre o Callosobruchus maculatus (Coleoptera: Bruchidae). Revista Caatinga, v.22, n.1, p.146150 , 2009. https://periodicos.ufersa.edu.br/index.php/caatinga/article $\underline{\text { /view/1009 }}$

Kotkar, H. M.; Mendki, P. S.; Sadan, S. V. G. S.; Jha, S. R.; Upasani, S. M.; Maheshwari, V. L. Antimicrobial and pesticidal activity of partially purified flavonoids of Annona squamosa. Pest Management Science, v.58, n.1, p.33-37, 2002. https://doi.org/10.1002/ps.414

Mazzonetto, F. E.; VendramiM, J. D. Efeito de pós de origem vegetal sobre Acanthoscelides obtectus (Say.) (Coleoptera: Bruchidae) em feijão armazenado. Neotropical Entomology, v.32, n.1, p.145-149. 2003. http://www.scielo.br/pdf/\%0D/ne/v32n1/15584.pdf

Panizzi, A. R.; Parra, J. R. P. Ecologia nutricional de insetos e suas aplicações no manejo de pragas. São Paulo: Malone, 391p. 1991.

Péret-Almeida, L. Curcuma longa L. Separação e caracterização de pigmentos curcuminóides, avaliação da atividade antimicrobiana, cultivo in vitro para o estabelecimento de gemas, calos, órgãos (raízes) e produção de metabólitos secundários. 2006. 136p. Tese (Doutorado em Ciência de Alimentos) - Universidade Federal de Minas Gerais. Belo Horizonte, 2006.

Potenza, M. R.; Arthur, V.; Felício, J. D.; Rossi, M. H.; Sakita, M. N.; Silvestre, D. F.; Gomes, D. H. P. Efeito de produtos naturais irradiados sobre Sitophilus zeamais Mots. (Coleoptera: 
Curculionidae). Arquivos do Instituto Biológico, v.71, p.477-484, 2004.

Procópio, S. O.; Vendramim, J. D.; Ribeiro Júnior, J. I.; Santos, J. B. Efeito de pós vegetais sobre Acanthoscelides obtectus (Say) e Zabrotes subfasciatus (Boh.) (Coleoptera: Bruchidae). Revista Ceres, v.50, n.289, p.395405, 2003. http://www.ceres.ufv.br/ojs/index.php/ceres/article/view/ $\underline{2877}$

Regnault-Roger, C. The potential of botanical essential oils for insect pest control. Integrated Pest Management Reviews, v.2, n.1, p.25-34, 1997.

https://link.springer.com/article/10.1023/A:10184722278 $\underline{89}$

Silva Neto, J. S. Atividades de formulações em pó provenientes de frutos de Piper tuberculatum sobre Zabrotes subfasciatus mantidos em grãos de feijão-fava. 2015. 66p. Dissertação (Mestrado em Agronomia) Universidade Federal do Piauí. Teresina, 2015. http://hdl.handle.net/123456789/341
Souza, A. L.; Dias, L. P.; Cardoso, J. R.; Nascimento, V. L. V. Bioatividade do extrato aquoso do açafrão (Curcuma longa L.) sobre o crescimento micelial de Colletotrichum lindemunthianum. Anais... Anais do $\mathbf{V}$ CONNEPI, Congresso de Pesquisa e Inovação da Rede Norte Nordeste de Educação Tecnológica 2010. IFAL, Maceió, 2010.

Vale, J. C.; Bertini, C.; Borém, A. Feijão-caupi do plantio à colheita. Viçosa, MG: Ed. UFV, 267p. 2017.

Vieira, P. C.; Fernandes, J. B.; Andrei, C. C. Plantas inseticidas. In: Simões, C. M. O.; Schenkel, E. P.; Gosmann, G.; Mello, J. C. P; Mentz, L. A.; Petrovick, P. R. Farmacognosia: da planta ao medicamento. 5 ed. Porto Alegre, RS: Ed. da UFSC, 2004, 1102p.

Vieira, P. C.; Mafezoli, J.; Biavatti, M. W. Inseticidas de origem vegetal. In: Ferreira, J. T. B.; Corrêa, A. G.; Vieira, P. C. Produtos naturais no controle de insetos. São Carlos: Ed. da UFSCar, v. 3, 2001, 176p. 